\title{
Probabilistic Load Flow Calculation Method of Containing Wind Farm Considering Grid Configuration Uncertainties
}

\author{
Hongsheng Su and Yan Yan \\ School of Automation and Electrical Engineering, Lanzhou Jiaotong University, \\ Lanzhou 730070, China \\ shsen@163.com
}

\begin{abstract}
Current probabilistic power flow calculation methods mostly consider the uncertainties of loads and the random failures of generators without thinking about the changing of the grids structure. Hence, this paper proposes a new probabilistic power flow calculation method comprehensively considering the influences of the uncertainties of wind farms, loads, generators, and grids structure on power flow calculation. The linear relationships are deduced between the nodes injection power and the branch active power as the circuits being at failures, and the cumulative probability distribution of each branches power flow is calculated by using semi-invariant and Gram-Charlier series expansion, and such that the complicated convolution operation is avoided. Combining compensation method and the conditional probability theory to deal with network structure changes of random factors, the paper establishes a probability flow calculation model comprehensively considering diverse factors such as random outputting power of the wind farms, random changes of the loads, and random failures of the generators, and the random variation of the grid structures and so on, the probability distribution function and probability density function of each branch can be quickly obtained by the model. Through the analysis on IEEE 14-node system, the uncertainty of grid structure has a remarkable effect on the probability distribution of the quantity to be solved. Hence, applying the proposed method can provide planners with more accurate and comprehensive information.
\end{abstract}

Keywords: Probabilistic power flow, semi-invariant, Gram-Charlier series, wind farm

\section{Introduction}

With the installed capacities increasing, wind farm connected to power grid generates more and more great influence on power flow distribution. Due to the inherent randomness and intermittence of wind speed, compared with traditional coal-fired power units, wind turbines possess different output characteristics, which cause the randomness of the wind generators on their own efforts, as a result, the wind farms connected to grids generates great influence on the distribution of active and reactive power currents, and voltage stability. Compared with the traditional power flow analysis method, the probability power flow analysis method can consider the randomness of the outputs of wind farms, better, and reflect the influence of the wind farm connected to grids on flow distribution of power grids more comprehensively. There are a lot of uncertainty factors existing in power system operation and planning stages, such as random change loads, and random failure generators, and etc. If we apply the determinacy calculation method to analyze the system status, a lot of calculations and scheme comparisons require to be conducted for many possible situations. This will cause a large amount of calculation quantity, and such that it is difficult to reflect the status of the system as a whole. However, the probability flow method could give a full comprehensive performance evaluation and make a quantitative analysis on the weak links of power grids for the 
entire power grid under various operation conditions by applying the probabilistic approaches to describe various uncertain factors and finally work out each state variable, such as branch flows and node voltages of probability statistical properties, e.g., the expected value, and variance and probability distribution function, and etc.

The domestic and foreign scholars have done a lot of research work since probability flow is proposed by Borkowska in 1974[1]. But general probabilistic power flow methods only consider random variation of the loads and random failure of the generators, and not conduct the random changes of grids structure[2,3]. As a fact, the changes of the grids structure have a remarkable impact on the systems power flow distribution. In [4] the branch fault is equivalently seen as the virtual power at both ends of the branch meeting 0-1 distribution, and the distribution of the discrete random variable is determined by Von Mises step function, and the probability distribution of voltage and branch current then is obtained through convolution operation with a continuous distribution of random variables. In [5] distribution coefficient method is used to consider the fault effects on the branch flow probability distribution of the system. In [6] a probability flow algorithm containing uniform flow controller (UPFC) is proposed, where the selection of the initial value of UPFC on convergence properties influence is discussed by use of UPFC equivalent power injection model, and the control role on circuits flow and nodes voltage are analyzed under the random faults of the generators and circuits.

\section{Model Description}

\subsection{Semi-invariant}

Definition1. Let $F(X)$ be probability distribution function(PDF) of random variable $X$, and $t$ be a real number with $\left|e^{i t x}\right|=1$, and then the function $g(x)=e^{i t x}=\cos t x+i \sin t x$ is integrable at interval $(-\infty,+\infty)$ on $F(X)$, and so the characteristic function(CF) of the real variable $t$ corresponding to $F(X)$ is described below [7, 8].

$$
\phi(t)=E\left(e^{i t x}\right)=\int_{-\infty}^{+\infty} e^{i t x} d F(x)
$$

If the $k$-order moment of CF exists, the formula above can then be expanded as Maclaurin series in a smaller neighboring field of $t$ by

And then

$$
\phi(t)=1+\sum_{u=1}^{k} \frac{a_{u}(i t)^{u}}{u !}+o\left(t^{k}\right)
$$

$$
\log \phi(t)=\sum_{u=1}^{k} \frac{\gamma_{u}(i t)^{u}}{u !}+o\left(t^{k}\right)
$$

where $\gamma_{u}$ is the semi-invariant[9].

For semi-invariant, there are two important properties below.

(1) If there are $n$ mutually independent random variables $x^{1}, x^{2}, \cdots, x^{n}$, and their respective $r$-order semi-invariant $\gamma_{u}^{1}, \gamma_{u}^{2}, \cdots, \gamma_{u}^{n}(u=1,2, \cdots, r)$ exist, and let $x$ be the sum of the $n$ random variables, and then the $r$-order semi-invariant of $x$ equals to the sum of the $r$-order semi-invariant of each independent random variables[10], namely,

$$
\gamma_{u}=\gamma_{u}^{1}+\gamma_{u}^{2}+\cdots+\gamma_{u}^{n}, \quad(u=1,2, \cdots, r)
$$

(2) If the random variable $y$ is the linear function of the random variable $x$, i.e., $y=a x+b$, and then $\gamma_{u}^{n}(u=1,2, \cdots, r)$ is the each-order semi-invariant of $x$, and then each-order semi-invariant of random variable $y$ is described by [11] 


$$
\gamma_{u}^{y}= \begin{cases}a \gamma_{1}^{x}+b & (u=1) \\ a^{u} \gamma_{u}^{x} & (u>1)\end{cases}
$$

Based on the above two features, we may use the semi-invariant feature to describe the random variable.

\subsection{Relationships between Semi-invariant and Moments}

Semi-invariant possesses very important properties, but from definition it can not be obtained directly for each-order semi-invariant of the random variables, and whose moments of the random variables are solved relatively easy below [12].

$$
\begin{gathered}
\gamma_{1}=\alpha_{1} \\
\gamma_{r+1}=\alpha_{r+1}-\sum_{j=1}^{r} C_{r}^{j} \alpha_{j} \gamma_{r-j+1} \\
\alpha_{r+1}=\gamma_{r+1}+\sum_{j=1}^{r} C_{r}^{j} \alpha_{j} \gamma_{r-j+1}
\end{gathered}
$$

where $\alpha_{r}$ is the origin moment, and $\gamma_{r}$ is the $r$-order semi-invariant, and $C_{r}^{j}$ is the binomial coefficients.

\subsection{Gram-Charlier Series}

For arbitrary random variable $X$, let its mean be $\mu$, and the standard deviation be $\sigma$, and its standardized random variable $\bar{X}$ can be written as

$$
\bar{X}=(X-\mu) / \sigma
$$

Let $F(x)$ be distribution function, and $f(x)$ be probability density function $(p d f)$ of the normalized random variable $X$, respectively, and then $f(x)=F^{\prime}(x)$. According to GrarnCharlier series expansion theory, $F(x)$ and $f(x)$ possess the following form[13].

$$
\begin{gathered}
F(x)=\Phi(x)+\left(\frac{c_{1}}{1 !}\right) \Phi^{\prime}(x)+\left(\frac{c_{2}}{2 !}\right) \Phi^{\prime \prime}(x)+\left(\frac{c_{3}}{3 !}\right) \Phi^{\prime \prime \prime}(x)+\cdots \\
f(x)=\phi(x)+\left(\frac{c_{1}}{1 !}\right) \phi^{\prime}(x)+\left(\frac{c_{2}}{2 !}\right) \phi^{\prime \prime}(x)+\left(\frac{c_{3}}{3 !}\right) \phi^{\prime \prime \prime}(x)+\cdots
\end{gathered}
$$

where $\Phi(x)$ and $\phi(x)$ respectfully are the cumulative distribution function and $p d f$ of the standard normal distributions, and the coefficient $c_{k}$ is defined by 


$$
c_{k}=(-1)^{k} \int_{-\infty}^{+\infty} H_{k}(x) f(x) d x \quad(k=1,2,3 \cdots, n)
$$

\section{Probabilistic Models of Wind Generator}

\subsection{Wind Speed Probability Model}

Currently, it often is considered to follow the Weibull model for the probability distribution of wind speed, whose $p d f$ is expressed below.

$$
f(v)=\frac{k}{c}\left(\frac{v-v_{0}}{c}\right)^{k-1} \exp \left[-\left(\frac{v-v_{0}}{c}\right)^{k}\right]
$$

where $v$ expresses the wind speed, and the parameters $k, c$, and $v_{0}$ are of the Weibull distribution ones, and $k$ denotes the shape parameter describing the characteristics of the wind speed distribution, and $c$ is the scale parameter meaning the size of the region average wind speed, and $v_{0}$ denotes the position parameters [14].

\subsection{Active Power Output Curve of Wind Generator}

The size of the outputting active power of wind turbines is mostly determined by wind speed value of wind generators position. Figure 1 shows a function relationship between wind speed and its output active power, which reflects the characteristics that the outputting active power of the wind generator varies with wind speed.

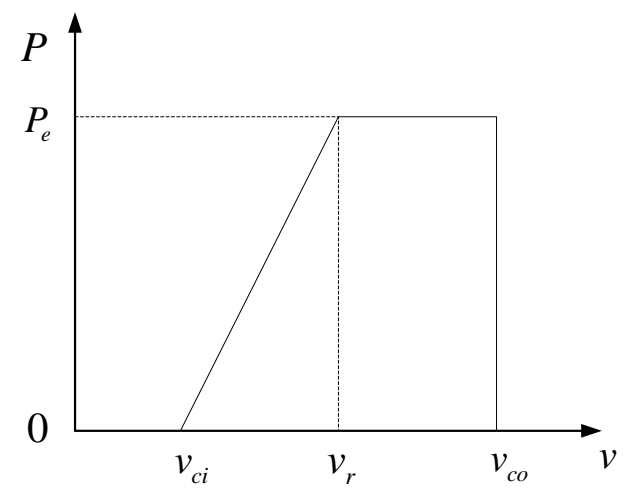

Figure 1. Wind Generator Active Power Output Curve

The relationship between wind speed $v$ and active power $P_{\mathrm{w}}$ of the wind turbines can be expressed by

$$
P_{\mathrm{w}}= \begin{cases}0 & v \leq v_{\mathrm{ci}} \\ k_{1} v+k_{2} & v_{\mathrm{ci}}<v<v_{\mathrm{r}} \\ P_{\mathrm{e}} & v_{\mathrm{r}} \leq v<v_{\mathrm{co}} \\ 0 & v \geq v_{\mathrm{co}}\end{cases}
$$

where $P_{\mathrm{e}}$ is the rated power of the generator, and $v_{\mathrm{ci}}, v_{\mathrm{r}}, v_{\text {co }}$ respectively are cut-in wind speed, and the rated speed, and cut-out wind speed, $k_{1}=P_{\mathrm{e}} / v_{\mathrm{r}}-v_{\mathrm{ci}}, k_{2}=-k_{1} v_{\mathrm{ci}}$. 


\subsection{Probability Distribution of Wind Generator Power}

The probability distribution model of the active power of the wind turbine can be obtained by the integral operation between the active power function of wind turbines and the PDF of wind speed [3].

From (14), as wind speed $v \leq v_{\mathrm{ci}}$ or $v \geq v_{\mathrm{co}}, P_{\mathrm{w}}=0$. At the moment, the PDF of wind active power can be obtained by integral calculation according to (13) and (14) as follows.

$$
f\left(P_{\mathrm{w}}\right)=\int_{0}^{v_{\mathrm{ci}}} f(v) d v+\int_{v_{\mathrm{co}}}^{+\infty} f(v) d v
$$

As wind speed $v_{\mathrm{r}} \leq v<v_{\mathrm{co}}$, then $P_{\mathrm{w}}=P_{\mathrm{e}}$. Likewise, we have

$$
f\left(P_{\mathrm{w}}\right)=\int_{v_{\mathrm{r}}}^{v_{\mathrm{co}}} f(v) d v
$$

As wind speed $v_{\mathrm{ci}}<v<v_{\mathrm{r}}$, we have $0<P_{\mathrm{w}}<P_{\mathrm{e}}$. In the same way, according to (13) and (14), we have

$$
F\left(P_{\mathrm{w}}\right)=\int_{0}^{v_{\mathrm{ci}}} f(v) d v+\int_{v_{\mathrm{ci}}}^{\frac{P_{\mathrm{w}}-k_{2}}{k_{1}}} f(v) d v
$$

\section{Probability Power Flow Analysis}

It is known to all that Newton-Raphson method is an effective method for nonlinear equations solution, it is for that applied to linearize power flow equations here. Firstly, the power flow equations are represented as polar form by [15]

$$
\left\{\begin{array}{l}
\Delta P_{i}=P_{i s}-U_{i} \sum_{j \in i} U_{j}\left(G_{i j} \cos \theta_{i j}+B_{i j} \sin \theta_{i j}\right) \\
\Delta Q_{i}=Q_{i s}-U_{i} \sum_{j \in i} U_{j}\left(G_{i j} \sin \theta_{i j}-B_{i j} \cos \theta_{i j}\right)
\end{array}\right.
$$

where $P_{i s}$ denotes the injected active power of the node $i$, and $Q_{i s}$ denotes the injected reactive power of the node $i, U_{i}$ and $U_{j}$ express the voltage values of the node $i$ and $j$, and $G_{i j}$ denotes the real part of the node admittance matrix, and $B_{i j}$ is the imaginary part, and $\theta_{i j}$ represents the phase difference between the node $i$ and the node $j$, and $j \in i$ means all the nodes that they must be attached to the node $i$.

Under normal operation, the formula (18) can be summarized by matrix form.

$$
W=f(X)
$$

where $\boldsymbol{W}$ denotes the injected active and reactive power vector of the mode, and $\boldsymbol{X}$ means the state vector composed of the node voltage and its phase, and $\boldsymbol{f}$ indicates flow equations.

In (19), $\boldsymbol{W}$ is a random variable, and can be written as

$$
W=\bar{W}+\Delta W
$$


where $\bar{W}$ is the expectation of the injected power, and $\boldsymbol{\Delta W}$ is a random disturbance. Similarly, the state variable $\boldsymbol{X}$ may also be expressed by

$$
X=\bar{X}+\Delta X
$$

where $\overline{\boldsymbol{X}}$ is the desired value of the state variable, and $\boldsymbol{\Delta} \boldsymbol{X}$ can be considered to be random variable following one distribution.

Substituting (20) and (21) into (19), and then expanding it at the desired operating point in the Taylor series, we obtain

$$
\bar{W}+\Delta W=f(\bar{X}+\Delta X)=f(\bar{X})+J \Delta X
$$

where

$$
\bar{W}=f(\bar{X})
$$

Hence, $\overline{\boldsymbol{X}}$ is the desired value of state variables under the role of the injected power desired value $\overline{\boldsymbol{W}}$ resolved by Newton-Raphson method, $\boldsymbol{J}$ is the last iterative Jacobian matrix using Newton method. (22) may be simplified as

$$
\Delta X=J^{-1} \Delta W=S \Delta W
$$

where $S$ is the sensitivity matrix.

The formula (24) shows a linear transformation of a random variable, where $\boldsymbol{\Delta} \boldsymbol{X}$ can be seen as a response to the random disturbance $\boldsymbol{\Delta W}$. Clearly, the probability distribution of $\boldsymbol{\Delta X}$ can be solved by the one of $\boldsymbol{\Delta W}$.

For branch flow equations, similar linear processing also can be done. branch flow equations under the form of polar coordinates can be written as

$$
\left\{\begin{array}{l}
P_{i j}=U_{i} U_{j}\left(G_{i j} \cos \theta_{i j}+B_{i j} \sin \theta_{i j}\right)-\frac{1}{k_{i j}} U_{i}^{2} G_{i j} \\
Q_{i j}=U_{i} U_{j}\left(G_{i j} \sin \theta_{i j}-B_{i j} \cos \theta_{i j}\right)+\frac{1}{k_{i j}} U_{i}^{2} B_{i j}-U_{i}^{2} b_{i j 0}
\end{array}\right.
$$

where $k_{i j}$ denotes the pu of branch transformation ratio, and $b_{i j 0}$ means half of the branches admittance.

Similar to the derivation process of (19), we obtain

$$
Z=g(X)
$$

where $\boldsymbol{Z}$ denotes the power vector of the branch flow, and $\boldsymbol{g}$ expressed the branch flow equations. Likewise, we have

$$
Z=\bar{Z}+\Delta Z
$$


where $\bar{Z}$ is the branch flow expectations, and $\boldsymbol{\Delta Z}$ is a random variable. Substituting (21) and (27) into (26), and then expanding it at the desired operating point in the Taylor series, we obtain

$$
\bar{Z}+\Delta Z=g(\bar{X}+\Delta X)=g(\bar{X})+G \Delta X
$$

Wherein

$$
\bar{Z}=g(\bar{X})
$$

Seen from (28), the matrix $\boldsymbol{G}$ can be obtain by the last Jacobian matrix in Newton iteration method. The (28) may be simplified as

$$
\Delta Z=G \Delta X=G J^{-1} \Delta W=G S \Delta W=T \Delta W
$$

where $\boldsymbol{T}$ is a transformation matrix.

Clearly, the probability distribution of $\boldsymbol{\Delta Z}$ can be solved by the one of $\boldsymbol{\Delta W}$.

\section{Randomly Switching On and Off Treatment for Branch}

Due to the external or own reasons, it is inevitable for electrical components in systems that the failure will appear. For transmission lines in primary system its fault situations are much complex and more due to the impact of field environment factors exerted in the wild. Random failure for system branches may apply compensation method to simulate. The compensation method refers to that when a branch switches off, the impact of which on system is simulated by the introduction of a power increment, i.e., the compensation power, at both ends of the breaking branch nodes. And thus, the original network can be considered to be unbreakable so as to the original admittance matrix can be applied without any modification, that is, the original network factor table can be used to solve the system state.

Definition 2 When one failure occurs in branch $l$ connected to the node $i$ and $j$, assuming that the branch $l$ does not switch off, only additional injection power increments $\Delta P_{i}$ and $\Delta P_{j}$ at two ends of the branch $l$, i.e., the node $i$ and $j$, are aroused. When the additional power increments is exactly equal to the original one that the branch $l$ switches on, and possesses opposite direction with it, we can then say that it is equivalent with the branch switching off.

As shown in Figure 2, the injected power via the node $i$ and $j$ respectively are $P_{i}+j Q_{i}$ and $P_{j}+j Q_{j}$ before the branch between $i$ and $j$ is switched off, two virtual injection powers to the ground are zero. After switching off, the virtual injection amount from the node $i$ and $j$ separately are $\Delta P_{i}+j \Delta Q_{i}$ and $\Delta P_{j}+j \Delta Q_{j}$, the branch power flow variations caused by it respectively are $\Delta P_{i j}+j \Delta Q_{i j}$ and $\Delta P_{j i}+j \Delta Q_{j i}$, and satisfy

$$
\begin{gathered}
\Delta P_{i}+j \Delta Q_{i}=\left(P_{i j}+\Delta P_{i j}\right)+j\left(Q_{i j}+\Delta Q_{i j}\right) \\
\Delta P_{j}+j \Delta Q_{j}=\left(P_{j i}+\Delta P_{j i}\right)+j\left(Q_{j i}+\Delta Q_{j i}\right)
\end{gathered}
$$




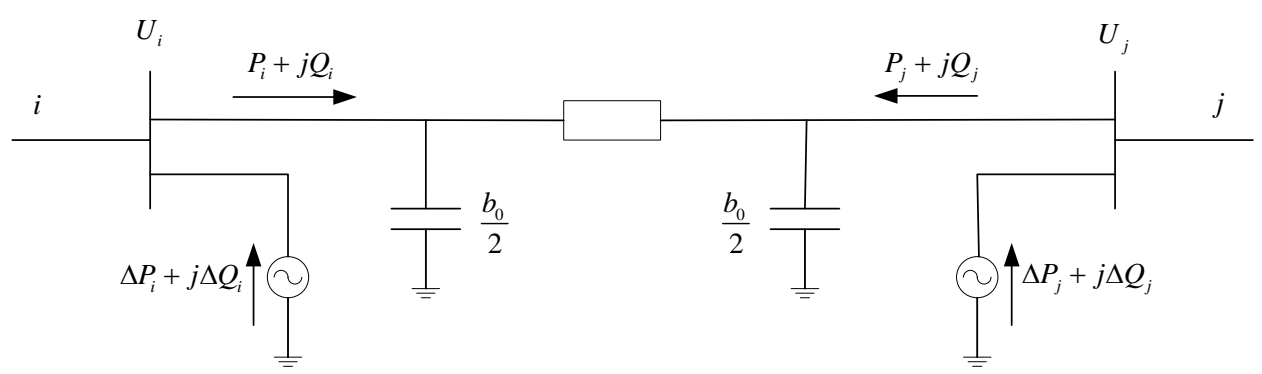

Figure 2. Branch Random Disconnection Mimic Diagram

After transformation on (31) and (32), we have

$$
\left[\begin{array}{l}
P_{i j} \\
Q_{i j} \\
P_{j i} \\
Q_{j i}
\end{array}\right]=\left[\begin{array}{c}
\Delta P_{i} \\
\Delta Q_{i} \\
\Delta P_{j} \\
\Delta Q_{j}
\end{array}\right]-\left[\begin{array}{c}
\Delta P_{i j} \\
\Delta Q_{i j} \\
\Delta P_{j i} \\
\Delta Q_{j i}
\end{array}\right]=\left[I_{4 \times 4}-T_{4 \times 4}\right]\left[\begin{array}{c}
\Delta P_{i} \\
\Delta Q_{i} \\
\Delta P_{j} \\
\Delta Q_{j}
\end{array}\right]
$$

where $I_{4 \times 4}$ is the 4-order unit matrix, $T_{4 \times 4}$ is a sub-array of the correlation matrix $T$ generated by deterministic flow equations of the reference state, and then according to disconnection situation the corresponding subarray $T_{4 \times 4}$ is formed, further. Similarly, we also can get a matrix with double and multiple wire breaking. $T_{4 \times 4}$ can be described below.

$$
T_{4 \times 4}=\left[\begin{array}{llll}
\frac{\partial P_{i j}}{\partial P_{i}} & \frac{\partial P_{i j}}{\partial Q_{i}} & \frac{\partial P_{i j}}{\partial P_{j}} & \frac{\partial P_{i j}}{\partial Q_{j}} \\
\frac{\partial Q_{i j}}{\partial P_{i}} & \frac{\partial Q_{i j}}{\partial Q_{i}} & \frac{\partial Q_{i j}}{\partial P_{j}} & \frac{\partial Q_{i j}}{\partial Q_{j}} \\
\frac{\partial P_{j i}}{\partial P_{i}} & \frac{\partial P_{j i}}{\partial Q_{i}} & \frac{\partial P_{j i}}{\partial P_{j}} & \frac{\partial P_{j i}}{\partial Q_{j}} \\
\frac{\partial Q_{j i}}{\partial P_{i}} & \frac{\partial Q_{j i}}{\partial Q_{i}} & \frac{\partial Q_{j i}}{\partial P_{j}} & \frac{\partial Q_{j i}}{\partial Q_{j}}
\end{array}\right]
$$

From (33) we obtain the injection amount of virtual power, the additional injection amount is then obtained of the nodes, and so branch wire breaking inject may be equivalent to the additional injected amount $\Delta P_{i}+j \Delta Q_{i}$ and $\Delta P_{j}+j \Delta Q_{j}$. Branches wire breaking is expressed by $0-1$ distribution table, where 0 represents the branch intact, and 1 for the branch breaking.

\section{Processing Model on Branch Correlation}

The branch correlation in system still contains the ones caused by season, geography, environment and other factors. It is difficult to quantify the correlation, generally, and is estimated by a statistical analysis and experience on spot. For conveniently analysis, in 
this article the correlation does not considered, and the involved one refers to that grid power flow redistribution is caused due to branch switching on and off.

Definition3 Let $l_{1}, l_{2}, \cdots, l_{n}$ represent $n$ breaches in system, and the random event $\bar{A}_{1}, \bar{A}_{2}, \cdots, \bar{A}_{n}$ represent the intact and normal operating of the corresponding branch, and $A_{1}, A_{2}, \cdots, A_{n}$ express the fault case for the corresponding branch, and then the probability of the event occurring can be described by

$$
P\left(A_{k}\right)=\operatorname{Fault}\left(l_{k}\right)(k=1,2, \cdots, n)
$$

where Fault $\left(l_{k}\right)$ represents the probability of branch failure.

The conditional probability $P\left(A_{k+1} \mid A_{k}\right)$ can be then worked out based on $P\left(A_{k}\right)$ and $P\left(A_{k+1}\right)$ and as well as their joint PDF, which can be used to describe the correlation between them. In this paper, in order to consider the issue conveniently, such an assumption is made that the line flow follows the normal distribution.

\section{Specific Applications on Semi-invariant}

Random disturbance of the injected power at each node is aroused by the node load randomness disturbance, and the random change of the generator outputs, and the compensation power caused by the random wire breaking, and random power outputs of wind turbines, which can be expressed by

$$
\Delta W=\Delta W_{g} \otimes \Delta W_{l} \otimes \Delta W_{y} \otimes \Delta W_{f}
$$

where $\Delta W_{g}$ is a random change of the generator nodes, and $\Delta W_{l}$ is a random change of the node loads, and $\Delta W_{y}$ is a random compensation power, and $\Delta W_{f}$ is the random power output of the wind generators.

The probability distribution can be then obtained by convolution operation of each state variable and branch power under each injected power known. However, its computation amount is quite large to use conventional convolution operation as the distribution of the random variables to solve. Hence, we use the semi-invariant and GramCharlier series expansion to replace conventional convolution operation to solve the distribution of random variables as follows.

Each-order semi-invariant of the injected power of the node can be obtained by use of semi-invariant additive property, and then from (36), we have

$$
\Delta W^{(k)}=\Delta W_{g}^{(k)}+\Delta W_{l}^{(k)}+\Delta W_{y}^{(k)}+\Delta W_{f}^{(k)}
$$

where $\Delta W_{g}^{(k)}, \Delta W_{l}^{(k)}, \Delta W_{y}, \Delta W_{f}^{(k)}$ respectively are semi-invariant of each node variable, and $k$ denotes $k$-order semi-invariant.

Applying another important property of semi-invariant, each-order semi-invariant on $\Delta X$ and $\Delta Z$ can be obtained by

$$
\begin{aligned}
\Delta X^{(k)} & =S_{0}^{(k)} \Delta W^{(k)} \\
\Delta Z^{(k)} & =T_{0}^{(k)} \Delta W^{(k)}
\end{aligned}
$$


where $S_{0}{ }^{(k)}$ and $T_{0}^{\left({ }^{k}\right)}$ are respectively formed by k-power of the elements in $S_{0}$ and $T_{0}$, that is, for any element $i$ and $j$, we have

$$
\begin{gathered}
S_{0}^{(k)}(i, j)=\left[S_{0}(i, j)\right]^{(k)} \\
T_{0}^{(k)}(i, j)=\left[T_{0}(i, j)\right]^{(k)}
\end{gathered}
$$

Therefore, after the each-order semi-invariant is calculated, the cumulative distribution functions on node voltage and branch power can be obtained by Gram-Charlier series expansion.

\section{Examples Analysis}

This paper takes IEEE 14-node system as an example, combining with the former analysis methods, and considers one wind power plant in western China connected to IEEE-14-node system through the node 14 as shown in Fig.3. The access wind farm contains 20 wind generators of each cell being $1 \mathrm{MW}$, and system parameters are made to be per unit referenced as 100MVA. For calculation purposes, this article assumes that loads power follows normal distribution and generators power output is $0-1$ distribution.

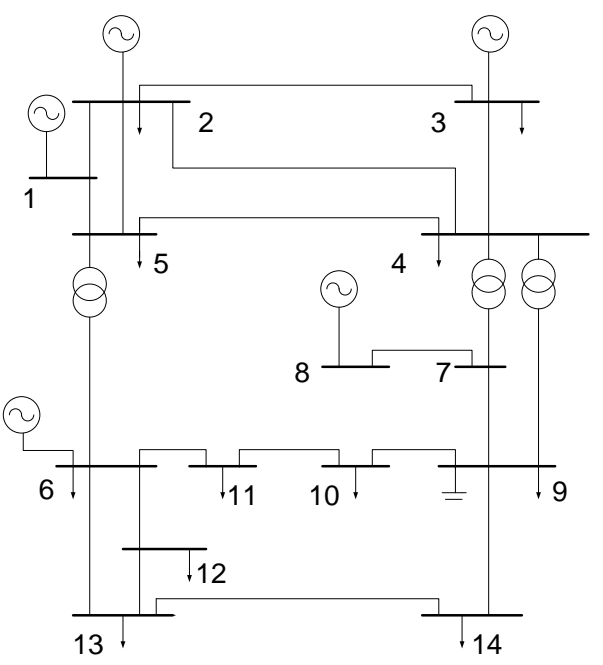

Figure 3. IEEE 14-node Grid Structure

(1) Figure 4 and Figure 5 respectively shows the cumulative probability distributions of the node voltages of the node 14 and the node 11 before and after the wind farm is connected, where the solid line represents the cumulative probability distribution that wind farm is not connected to the grid, and the dashed line represents the ones that the wind farm is connected the grid. 


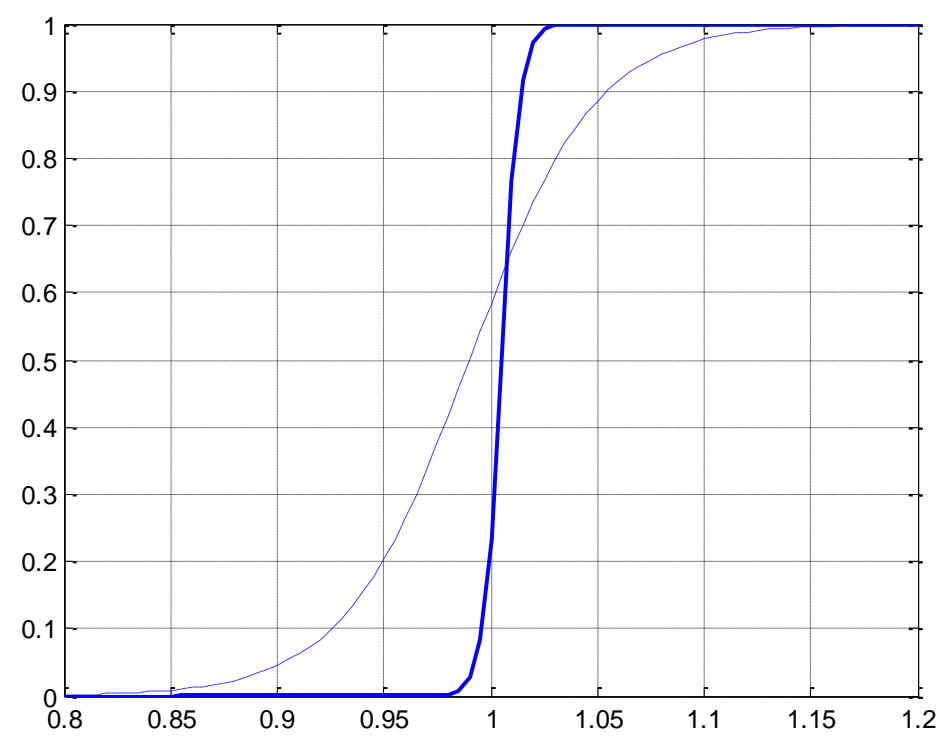

Figure 4. Cumulative Probability Distribution Diagrams on 14-node Voltage

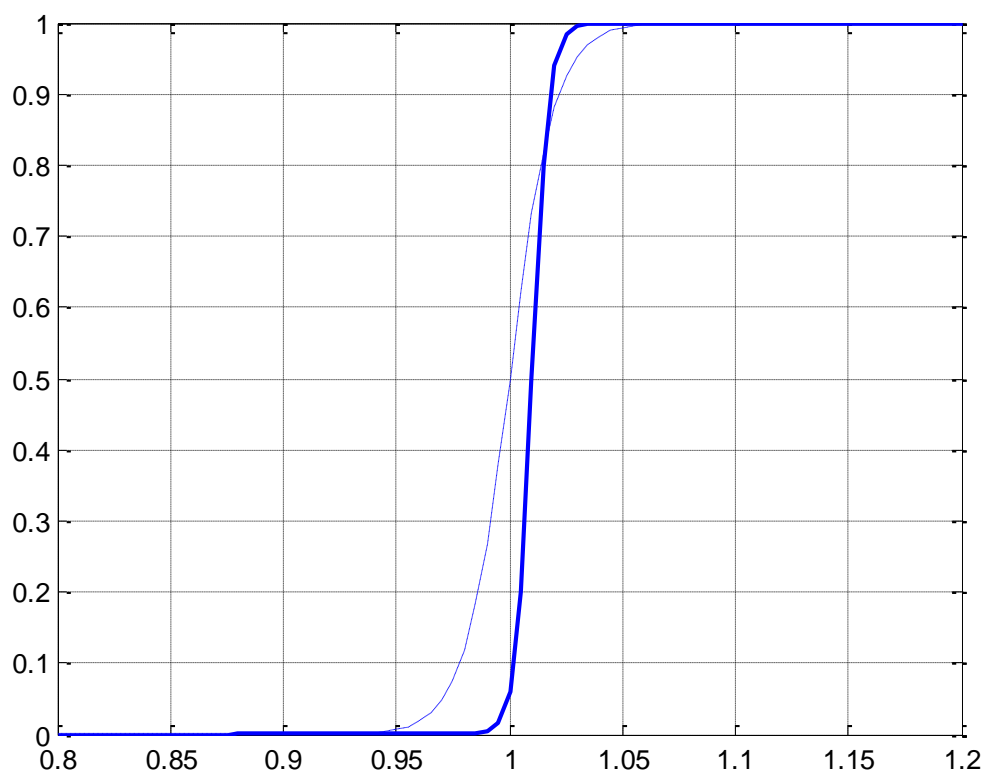

Figure 5. Cumulative Probability Distribution Diagrams on 11-node Voltage

Seen from the two Figures, apparently, after wind farm is connected the node voltages have greater fluctuation, which means that the wind farm access has a certain impact on the system bus voltage. The main reason lies in the randomicity of wind farm output power.

(2) The cumulative probability distribution of the nodes voltage is respectively shows in Figure 6, Figure 7, Figure 8, and Figure 9 for these randomly selected nodes such as 13, 9, 11 , and 4 , where the dashed lines denote cumulative probability distribution of the node voltage without considering correlation between the branches, and the solid lines indicate the one under it being considered. 


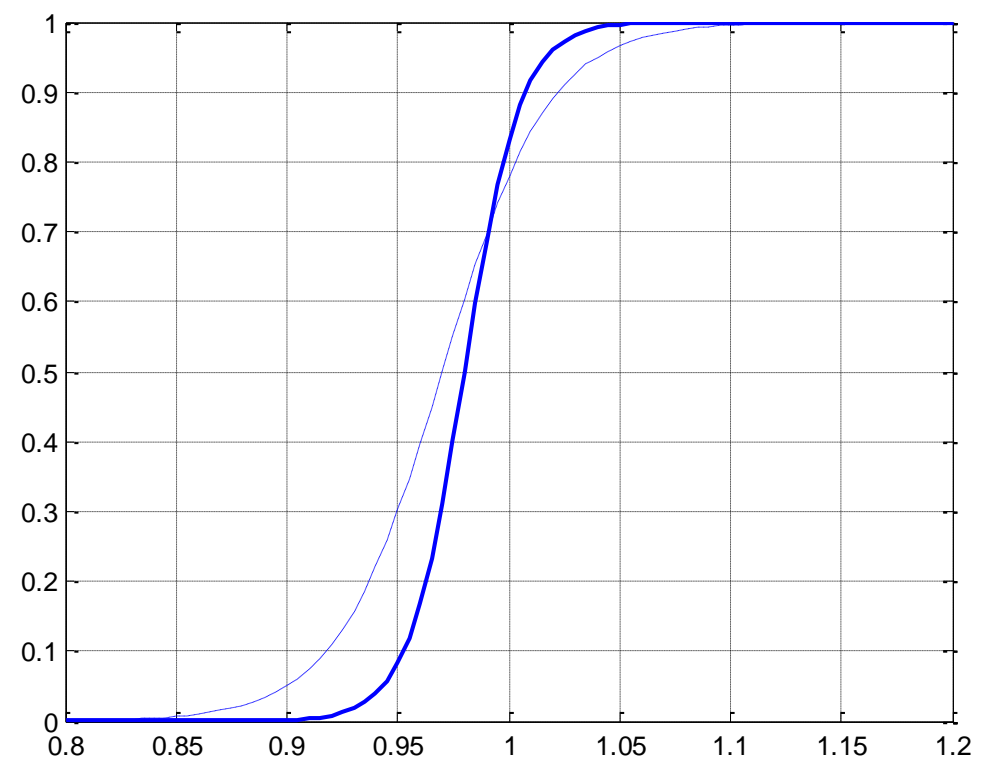

Figure 6. Cumulative Probability Distribution Diagrams on 13-node Voltage

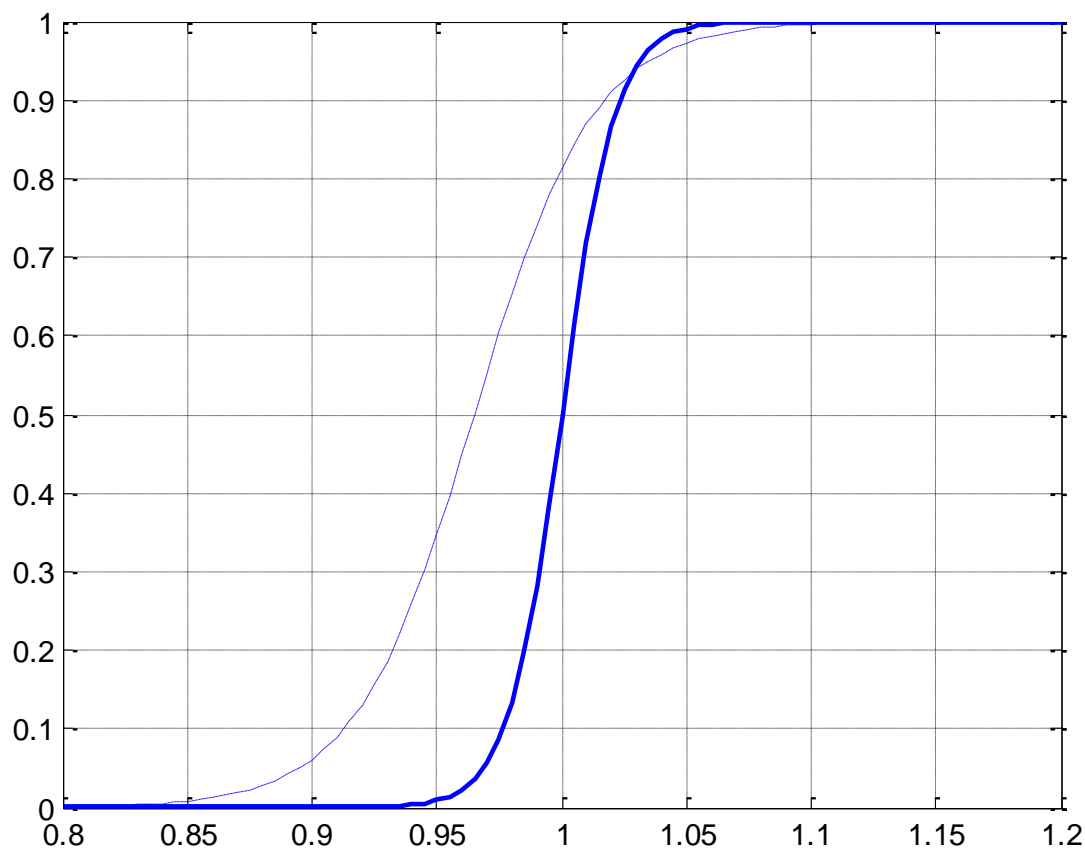

Figure 7. Cumulative Probability Distribution Diagrams on 9-node Voltage 


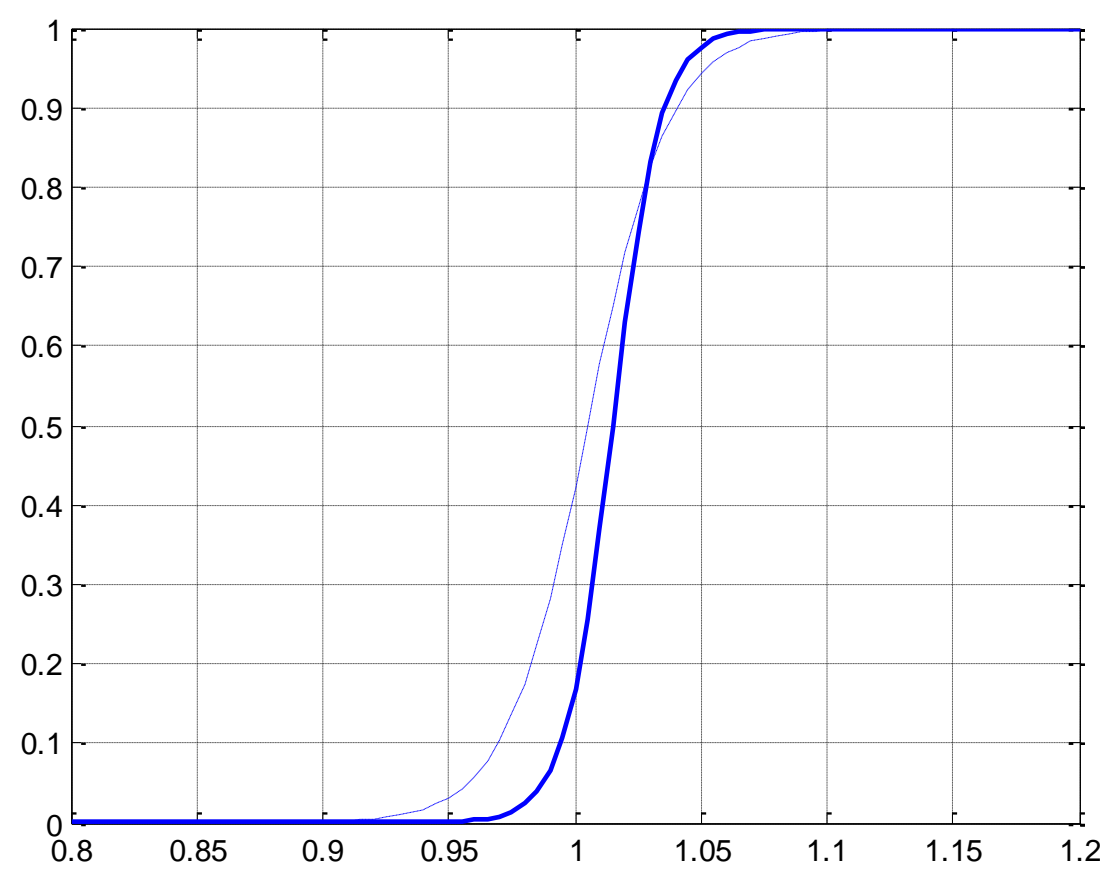

Figure 8. Cumulative Probability Distribution Diagrams on 11-node Voltage

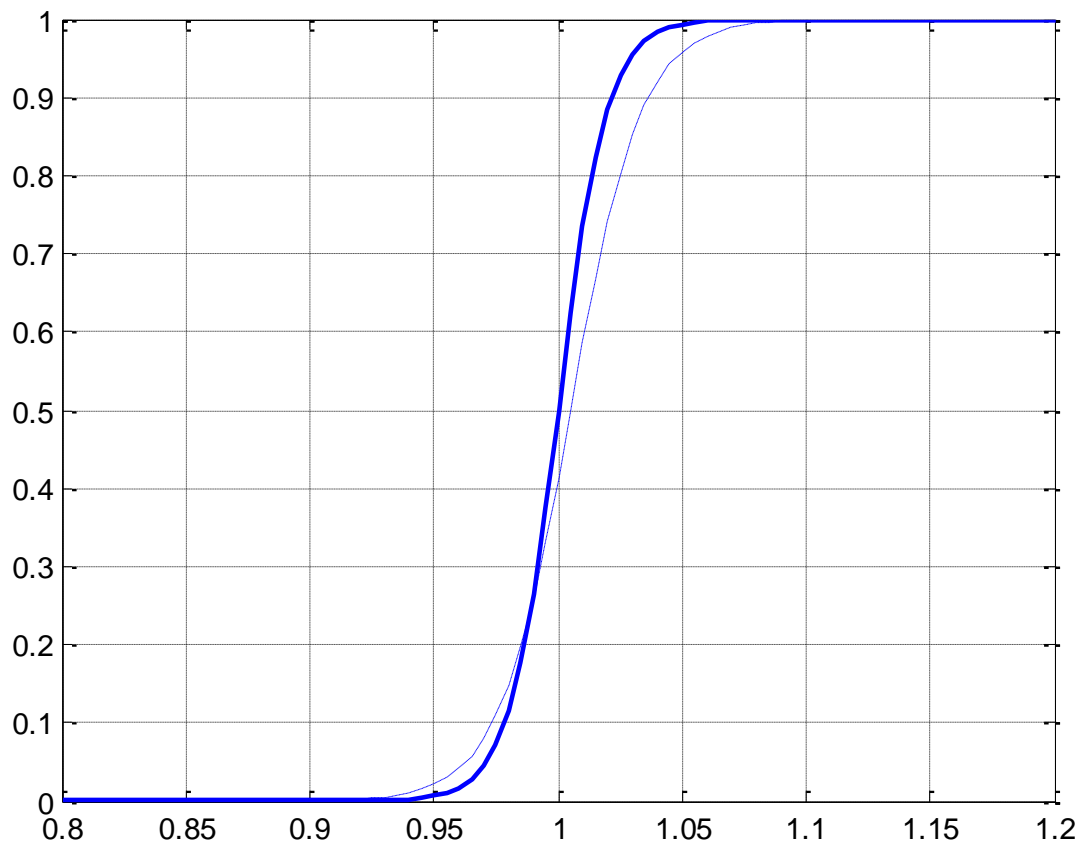

Figure 9. Cumulative Probability Distribution Diagrams on 4-node Voltage

Seen from the above Figures, there are a more obvious changes occurring in cumulative probability distribution of the node voltage when considering the correlation between the branches, and the fluctuation ranges of the nodes voltage increase so that more out-oflimit probabilities occurs, which can seen in Table 1. 
Table 1. Out-of- limit Probability of the Node Voltage

\begin{tabular}{ccc}
\hline Node number & Excluding correlation & Consider the correlation \\
\hline 13 & $8.22 \%$ & $32.16 \%$ \\
9 & $1.76 \%$ & $36.52 \%$ \\
11 & $2.02 \%$ & $8.62 \%$ \\
4 & $1.16 \%$ & $7.45 \%$ \\
\hline
\end{tabular}

From Table 1, there is more out-of-limit probability occur when considering the correlation between the branches for the 13-node voltage compared with no-considering, the former arrives at $32.16 \%$, and the latter is only $8.22 \%$. Another an example is the node 9 of its out-of-limit probabilities being $36.52 \%$ and $1.76 \%$ under the two conditions. For the node 11 and 4, their voltage fluctuations are relatively smaller, the main reason lies in that the node 13 and node 9 possess closer electrical distance to wind farm compared with the node 11 and node 4 .

\section{Conclusion}

This paper proposes a comprehensive probability power flow calculation method when considering diverse factors, simultaneously, such as wind farm random outputs, and load random changes, and generator random failures, and as well as random changes of network structure. The main conclusions are below.

(1) Compensation method is applied to deal with network structure changes aroused by circuits failure randomly, and linear relationship is deduced between the injected power at each node and branch power each branch when the circuit being in failure, and thus the complicated convolution operation is avoided by semiinvariant method and Gram-Charlier expansion to solve the probability distribution of each branch power flow.

(2) Probability power flow calculation model is obtained considering random loads variation, and the random failures of the generators, and random network structure changes by using conditional probability theory to deal with the any uncertainties of grids structure changes caused by any branches are in failure.

(3) Wind power generation brings system a certain fluctuation due to its own random outputs, which makes the system voltage stability, and security, and normal operation influenced to certain extent.

(4) The simulation example shows that the calculation results are more close to practice when considering the uncertainty of the network structure, and possesses practical applications prosperity.

\section{References}

[1] B. Borkowska, "Probabilistic load flow", IEEE Transactions on Power Apparatus and Systems, vol. 3, no. 3, (1974), pp.751-755.

[2] P. W. Sancer, and G. T. Heydt, "A generalized stochastic power flow algorithms", IEEE Summer Meeting, (1978) July 16-21; Los Angeles, California, USA.

[3] G. T. Heydt, "Stochastie Power Flow Calculations", (1975) July; IEEE Summer power Meeting, SanFraneiseo, California, USA.

[4] L. da Silva A. M. and V. Arienti, "Probabilistic load flow considering dependence between input nodal powers", IEEE Transactions on Power Appartus and Systems, vol. 103, no. 6, (1984), pp. 1521-1535.

[5] L. da Silva, A. M. and V. Arienti, "Probabilistic load flow by a multilinear simulation algorithm", IEE Proceedings: Generation, Transmission \& Distribution, vol. 137, no. 4, (1990), pp. 272-280.

[6] M. Ding, S. Li, and K. Huang, "Probabilistic load flow analysis based on Monte-Carlo simulation", Power System Technology, vol. 25, no. 11, (2001), pp. 11-16.

[7] R. N. Allan, A. M. L. Silva da and R. C. Burchett, "Evaluation methods and accuracy in probabilistic load flow solutions", IEEE Transactions on Power Appartus and Systems, vol. 100, no. 5, (1981), pp. 2534-2547. 
[8] S. Chun-Lien, "Probabilistic load flow computation using point estimate method", IEEE Transactions on Power Systems, vol. 20, no. 4, (2005), pp.1843-1851.

[9] J. M. Morales and J. Perez-Ruiz, "Point estimate schemes to solve the probabilistic power flow", IEEE Transactions on Power Systems, vol. 22, no. 4, (2007), pp. 1591-1605.

[10] L. Dong, C. Zhang, Y. Yang and P. Zhang, "Improvement of probabilistic load flow to consider network configuration uncertainties", in Proceedings of Power and Energy Engineering Conference, AsiaPacific(APPEEC), (2009) March 28-31; Wuhan, China.

[11] L. Dong and W. Cheng, "A probabilistic load flow method with consideration of random branch outages and its application", in Proceedings of Power and Energy Engineering Conference, AsiaPacific(APPEEC), (2009) March 28-31; Chengdu, China.

[12] J. Dai, H. Wang, S. Zhou, Z. Lu and L. Zhu, "A study on probabilistic of voltage instability based on stochastic characteristic of loading margin index", Proceedings of CSEE, vol. 26, no. 13, (2006), pp. 2630.

[13] X. Yu, H. Jia and J. Chen, “A preliminary research on power system cascading failures”, Power System Technology, vol. 30, no. 13, (2006), pp. 20-25.

[14] Y. Song and C. Wang, "A probabilistic risk assessment method for cascading failure of power system", Proceedings of CSEE, vol. 29, no. 4, (2009), pp. 27-33.

[15] X. Wang and X. Wang, "Probabilistic load flow analysis in power system", Journal of Xi'an JiaoTong University, vol. 22, no. 2, (1988), pp.

\section{Authors}

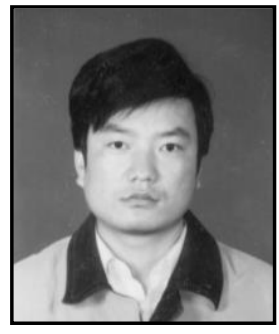

Hongsheng Su, he obtained his Master in Traffic Information Engineering and Control, Lanzhou Jiaotong University in 2001. He acquired his $\mathrm{PhD}$ in Power Systems and Its Automation, Southwest Jiaotong University. Now he is serving as a full-time professor at school of Automation and Electrical Engineering, Lanzhou Jiaotong University. His research interest includes System Security and Reliability, Intelligent Control, Power Systems and Its Automation, and etc.

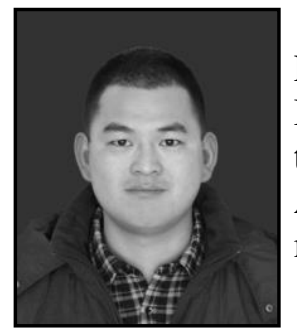

Yan Yan, he obtained his Bachelor in Lightning Protection Science and Technology, Nanjing University of Information Science and Technology. Now he is serving as a fulltime graduate student at school of Power Systems and Its Automation, Lanzhou Jiaotong University. His research interest mainly includes Probabilistic power flow calculate. 
International Journal of Security and Its Applications

Vol.9, No.8 (2015) 